\title{
Barcelona, de ciudad con turismo a ciudad turística. Notas sobre un proceso complejo e inacabado
}

\author{
Francesc López Palomeque
}

Universitat de Barcelona. Departament de Geografia Física i Anàlisi Geogràfica Regional lopez.palomeque@ub.edu

Recepción: marzo de 2015

Aceptación: junio de 2015

\section{Resumen}

El artículo constituye un relato explicativo sobre el proceso de conversión de Barcelona en una ciudad turística, particularmente a lo largo de las dos últimas décadas. Se aportan datos evolutivos de los distintos componentes del sistema turístico y se contextualiza el significado de las actividades turísticas en las estructuras de la ciudad y en su funcionamiento. Dicho proceso ha sido complejo y se muestra inacabado. Se parte de dos premisas específicas: a) son diversos los factores que explican el origen y el desarrollo del proceso, y entre ellos destaca el papel de la gestión pública del turismo, de su modelo de promoción y fomento, que es valorado como un modelo de éxito; $b$ ) en su nuevo estatus como ciudad turística, Barcelona tiene el reto de diseñar un modelo de gestión de la ciudad (turística) basado en la relación turismo-ciudad que prevea minimizar los conflictos y favorecer las sinergias.

Palabras clave: Barcelona; turismo; ciudad; modelo; gestión; destino turístico.

Resum. Barcelona, de ciutat amb turisme a ciutat turística. Notes sobre un procés complex $i$ inacabat

L'article constitueix un relat explicatiu sobre el procés de conversió de Barcelona en una ciutat turística, particularment al llarg de les dues últimes dècades. S’aporten dades evolutives dels diferents components del sistema turístic i es contextualitza el significat de les activitats turístiques en les estructures de la ciutat i en el seu funcionament. Aquest procés ha estat complex i es mostra inacabat. Es parteix de dues premisses específiques: a) són diversos els factors que expliquen l'origen i el desenvolupament del procés, i entre ells destaca el paper de la gestió pública del turisme, del seu model de promoció i foment, que és valorat com un model d'èxit; $b$ ) en el seu nou estatus com a ciutat turística, Barcelona té el repte de dissenyar un model de gestió de la ciutat (turística) basat en la relació turisme-ciutat que prevegi minimitzar els conflictes i afavorir les sinergies.

Paraules clau: Barcelona; turisme; ciutat; model; gestió; destinació turística. 
Résumé. Barcelone, d'une ville avec du tourisme à une ville touristique. Notes sur un processus complexe et inachevé

Cet article constitue un récit explicatif du processus de transformation de Barcelone en une ville touristique, particulièrement tout au long des deux dernières décennies. Des données évolutives issues des composantes du système touristique y sont apportées ainsi qu'une mise en contexte du signifié des activités touristiques dans les structures de la ville et dans son fonctionnement. Ce processus complexe et n'est pas achevé. Il est issu de deux prémisses spécifiques: $a$ ) Les facteurs expliquant l'origine et le développement du processus sont divers. Parmi ces processus, le rôle de la gestion publique du tourisme et son modèle de promotion, valorisé comme un modèle de réussite sont particulièrement importants; b) Par son nouveau statut social de ville touristique, Barcelone se donne le défi de concevoir un modèle de gestion de la ville (touristique) fondé sur la relation ville-tourisme, et qui vise à minimiser les conflits et favoriser les synergies.

Mots-clés: Barcelone; tourisme; ville; modèle; gestion; destination touristique.

Abstract. Barcelona, from a city with tourism to a tourist city. Notes on a complex and unfinished process

This article provides an explanatory account of the process to transform Barcelona into a tourist city, particularly over the last two decades. Evolutionary data of the various components of the tourism system are provided and the meaning of tourism activities is contextualized in the structures of the city and its operation. This process has been complex and remains unfinished. The article is based on two specific premises: $a$ ) the origin and development of the process can be explained by several factors, among them the role of public tourism management and its successful promotion and development model; $b$ ) due to Barcelona's new status as a tourist city, it faces the challenge of designing a management model of the (tourist) city based on the relationship between tourism and city, which involves minimizing conflicts and fostering synergies.

Keywords: Barcelona; tourism; city; model; management; tourist destination.

\section{Sumario}

\section{Presentación}

2. Factores del desarrollo turístico de Barcelona

3. Datos básicos de la oferta y la demanda: evidencias del desarrollo del turismo

4. Distribución espacial de la actividad turística: los cambios en el mapa turístico
5. Articulación del turismo en las estructuras preexistentes: afectaciones del turismo en la ciudad

6. Actores del proceso y protagonismo de la gestión pública del turismo 7. A modo de resumen y conclusiones Referencias bibliográficas 


\section{Presentación}

Barcelona está de moda. Es una afirmación recurrente en los medios de comunicación y referida a todos los ámbitos, en los que destaca su proyección internacional. Obviamente, esta condición no se ha alcanzado por casualidad, y en este estatus el turismo tiene mucho que ver. A priori, el papel del turismo no debería de extrañar, puesto que Barcelona es hoy día una ciudad turística y un destino urbano de primer orden a escala internacional, resultado de un largo proceso de evolución de las actividades turísticas. La relativa novedad deriva de la intensificación del proceso en las dos últimas décadas (perspectiva temporal) y de los cambios en la ciudad (dinámicas urbanas), que conforman la doble realidad señalada: destino de primer orden y ciudad turística (López Palomeque, 2009).

En relación con la perspectiva temporal, con la evolución de la ciudad, la situación actual es nueva. Barcelona ha pasado de ser una ciudad con turismo a ser una ciudad turística. En los años ochenta y noventa, la ciudad disponía de un sistema turístico eficaz, pero el conjunto de infraestructuras y actividades turísticas tenía una dimensión moderada en términos comparados con otras ciudades y, por otra parte, su peso en las estructuras de la ciudad era de carácter complementario.

En relación con la dinámica interna de la ciudad, con su metabolismo físico y social, en el último periodo han aparecido nuevos elementos y relaciones que modifican los componentes y las relaciones preexistentes. El dinamismo actual de la ciudad está marcado en buena parte por el grado de desarrollo alcanzado por las actividades turísticas, que se valora como un modelo de turismo urbano de éxito (Font y Sánchez, 2014; Serrano y Figueroa, 2014), y por el proceso de articulación del turismo en las estructura preexistentes. La relación turismo-ciudad no está exenta de conflicto e incluso, dada la visibilidad y la naturaleza de sus expresiones, la asociación turismo-conflicto ha emergido con fuerza en los debates sobre el modelo turístico de Barcelona, y ha dado paso a la aparición de posiciones de turismofobia que se contraponen al discurso de relación sinérgica, hasta ahora más generalizado, y de euforia.

Barcelona es uno de los destinos urbanos más destacados de Europa por el volumen de su capacidad de alojamiento y por el flujo de viajeros y pernoctaciones realizadas en sus hoteles. La superan seis u ocho ciudades, pero todas ellas son capitales de estado. En la clasificación de 2013 de European Cities Marketing (2014), Barcelona ocupa el quinto lugar de acuerdo con el número de viajeros en hoteles (7,5 millones), y sin contar las capitales políticas de los estados se sitúa en la primera posición de manera indiscutible (Londres, 28,5 millones; París, 15,6; Berlín, 11,3; Roma, 10,1). En el contexto de Cataluña, desde finales de los noventa, Barcelona es el municipio con mayor capacidad hotelera. En 1990 la oferta de la ciudad solo era de 18.569 plazas, en 2000 alcanzó la cifra de 31.338 plazas y en 2013 el censo registró 365 hoteles y 67.567 plazas, el 22\% del total de Cataluña. La ciudad, pese a los años de crisis económica, continúa batiendo récords en cuanto a los parámetros básicos 
del fenómeno turístico, tal como demuestran las cifras que se hacen públicas en los balances anuales, con crecimientos liderados por el dinamismo de la celebración de eventos y congresos y la consolidación de los cruceros (Garay y Cànoves, 2012).

Además de ser un destino turístico a escala internacional, Barcelona es una ciudad turística: el turismo ha superado la situación inicial de actividad complementaria y se ha convertido en un componente estructural de su sistema productivo, de su metabolismo, que marca su cotidianidad, que no deja indiferente a los ciudadanos y que ha configurado la imagen de la ciudad por la que se la reconoce internacionalmente. El grado de desarrollo de las actividades turísticas ha trascendido el sector y afecta a todos los componentes de la ciudad. El debate sobre el turismo en Barcelona está presente en los medios de comunicación, en la agenda de las instituciones, en el debate político, en la opinión y percepción de los barceloneses, en el portafolio de los empresarios e inversores y en la mirada y el imaginario de los viajeros. ¿Cómo se ha llegado a esta situación?

El objetivo de este artículo es ofrecer un relato explicativo sobre el proceso de conversión de Barcelona en una ciudad turística, particularmente a lo largo de las dos últimas décadas, aportando los datos evolutivos de los distintos componentes del sistema y contextualizando su significado en el marco de las estructuras y del funcionamiento de la ciudad. Dicho proceso ha sido complejo (naturaleza compleja del fenómeno turístico; funcionalidad preexistente y cambios de una ciudad de gran dimensión) y se muestra inacabado (en el marco de la consideración de las fases evolutivas en turismo y de los cambios del modelo de ciudad). Se parte de dos premisas específicas: a) son diversos los factores que explican el origen y el desarrollo del proceso, entre los que destaca el papel de la gestión pública del turismo; $b$ ) en su nuevo estatus de ciudad turística, Barcelona tiene el reto de asumir el paso de la gestión del turismo (modelo de gestión de la promoción y fomento del turismo) a la gestión de la ciudad (turística), con la adecuación del organigrama del gobierno de la ciudad a la nueva realidad.

En estas notas sobre el proceso se entiende por ciudad turística, en el marco conceptual de los estudios urbanos, aquella que incorpora las actividades de producción y consumo turísticos como una de sus funciones estructurales, debido a la relevancia y al significado que el turismo adquiere en sus distintos componentes y ámbitos (morfológico o constructivo, funcional o relacional —uso económico o social-, simbólico e institucional). No hay que olvidar, sin embargo, que Barcelona, como otras grandes ciudades, se caracteriza por la diversidad de sus funciones urbanas y, en este sentido, Gausa et al., (2010: 112) caracterizan Barcelona, desde 1992, como ciudad global y relacional y ciudad turística y atractiva.

El proceso específico de conversión de Barcelona en ciudad turística se inserta en la evolución histórica del turismo en la ciudad. Es su última etapa. El objetivo y las premisas que se plantean parten de una simplificación del proceso histórico del turismo en la ciudad, de su temporalización en etapas y 
periodos. En este ejercicio de simplificación se considera que en los primeros años noventa (hace veinticinco años) se inicia una etapa con diversos acontecimientos e hitos destacados, de inflexión y de ruptura, en la progresiva construcción histórica de Barcelona como destino, en la creación del sistema turístico de la ciudad, que finalmente ha conducido a su conversión en ciudad turística. Se identifican dos referencias principales: por una parte, los Juegos Olímpicos de 1992, que con todas sus repercusiones, por ejemplo el proceso producción de imagen en la Barcelona olímpica (Benach, 1993), favoreció situar a la ciudad en el mapa mundial; y, por otra parte, la creación del consorcio Turisme de Barcelona, que supuso un cambio en el modelo de gestión de la promoción y el fomento del turismo. La premisa considera que en esos años se conformó dicho sistema, que ha funcionado de manera eficaz. Y la premisa prevé, asimismo, la afirmación de que, con el paso del tiempo, de manera progresiva Barcelona se ha convertido en ciudad turística en los términos antes señalados. El análisis de la ciudad y la valoración del rol del turismo así lo confirman.

\section{Factores del desarrollo turístico de Barcelona}

El análisis convencional de los factores del desarrollo turístico de un destino considera, en un primer nivel, la diferenciación entre factores internos y factores externos. En esta ocasión, a partir de este esquema analítico inicial, interesa considerar el proceso general de eclosión del turismo urbano en las dos últimas décadas como contexto en el que se inserta el caso de Barcelona. Por otra parte, y siguiendo con la terminología analítica, por factores internos se entienden las circunstancias del propio lugar (Barcelona) que han favorecido su desarrollo turístico.

\subsection{La eclosión del turismo urbano}

El turismo urbano es un fenómeno complejo y se presenta desde finales de los años ochenta como uno de los sectores turísticos con demandas emergentes. El turismo en la ciudad es un fenómeno antiguo, pues el propio origen del turismo se encuentra en la atracción de la ciudad, pero tanto la dimensión que ha alcanzado hoy como la forma en que se manifiesta permiten hablar de un estallido reciente del turismo urbano. La ciudad de Barcelona ha vivido un gran desarrollo de las actividades turísticas y se puede considerar un ejemplo de esta evolución generalizada que se observa en la mayor parte de las ciudades medianas y grandes de Europa y de todo el mundo.

Son varias las razones que pueden explicar la expansión del turismo urbano, tanto desde el punto de vista de la demanda como de la oferta. Se pueden identificar al menos cuatro razones que fundamentan la actualidad y el interés del binomio turismo-ciudad y la expansión del turismo urbano: $a$ ) el cambio de paradigma turístico, que por sus características favorece al turismo urbano; b) las estrategias de desarrollo de las actividades turísticas en las ciudades y la búsqueda de la eficacia del sistema turístico de cada núcleo urbano; $c$ ) el 
desarrollo del turismo como estrategia para definir el modelo de ciudad, del presente y del futuro, en la nueva dialéctica de los procesos de producciónconsumo y del cambio de significado del espacio urbano; y d) el turismo como estrategia para reforzar la función internacional y para reforzar la ventaja en el escenario de competencia entre grandes ciudades en un mundo globalizado. Vera et al. (2013) abordan de manera más completa y detallada las causas y las grandes tendencias que impulsan y acompañan la eclosión del turismo urbano y metropolitano.

\subsection{Fundamentos del turismo de la ciudad}

Barcelona presenta los fundamentos básicos que caracterizan el turismo urbano: ciudad con patrimonio histórico y monumental singular, equipamiento y dinámica cultural destacada, capital económica y turismo de negocios que la ha destacado como ciudad de ferias y congresos, plaza comercial de gran atracción, ciudad universitaria, capital deportiva, centro de atracción de servicios especializados - entre otras funciones terciarias - y la oferta del frente marítimo recuperado, que, además de disponer de una amplia oferta de servicios lúdicos y recreativos, comprende cuatro kilómetros de playas. La Ciudad Condal se beneficia, además, de unas circunstancias que amplían su capacidad de atracción actual y futura: 1) su localización y sus condiciones ambientales (entorno mediterráneo y la mediterraneidad); 2) la celebración de los Juegos Olímpicos de 1992 y sus repercusiones en distintos órdenes; 3) la conformación definitiva de Barcelona como metrópolis mediterránea de primer orden y como ciudad internacional; 4) Barcelona es la capital de una región turística (Cataluña) de primer orden y entre ambas se establecen sinergias; y 5) la decidida gestión de la Administración pública en favor del turismo.

Situados en un contexto más reciente, en las dos últimas décadas, el desarrollo turístico de Barcelona se ha sustentado en las claves que explican tradicionalmente el éxito de los destinos urbanos citados en el punto anterior. En el haber de Barcelona se constata: $a$ ) ampliación y mejora de las infraestructuras de transporte y comunicación (creación de la nueva Terminal 1 del aeropuerto en 2009; línea del AVE, etc.); b) el reforzamiento de su imagen como ciudad con un rico patrimonio cultural y monumental en el que destaca la obra de Gaudí como máximo foco de atracción; $c$ ) el incremento de la oferta cultural y museística (en las dos últimas décadas se ha abierto o reformado un número destacado de equipamientos culturales y museos, los últimos el Born Centre Cultural en 2014 y el Museo del Diseño en 2015); d) el refuerzo de la capacidad de atracción en el segmento de turismo de reuniones (congresos, convenciones, incentivos y actividad ferial); e) la consolidación como plaza fuerte en los itinerarios de los cruceros mediterráneos; $f$ ) sus equipamientos deportivos, capacidad de organización de eventos de competiciones y el rol del FC Barcelona; $g$ ) su oferta comercial, que ha consolidado el segmento del turismo de compras; $y$, entre otros, h) la emergencia de la gastronomía. 
El atractivo de Barcelona no se fundamenta solamente en aquellos recursos y productos tangibles, y de manera más selectiva en aquellos iconos materiales que han ido conformando el imaginario social sobre la ciudad. Existen otros atractivos o iconos intangibles que tienen un papel muy destacado en la creación de la imagen de Barcelona. Se trata de la percepción de Barcelona como ciudad abierta, ciudad tolerante, ciudad amable. Atributos que se asocian a la ciudad y que, como tales, son percibidos por los ciudadanos de todo el mundo.

\section{Datos básicos de la oferta y la demanda: evidencias del desarrollo del turismo}

El impulso turístico inducido por los Juegos Olímpicos y la fuerte expansión de los últimos años pese a los efectos de la crisis han permitido que la capacidad de alojamiento hotelero de la ciudad se haya incrementado notablemente en los últimos veinte años y haya pasado de 18.569 plazas en 1990 a 67.567 en 2013 (Tabla 1). El censo hotelero de la ciudad es el resultado de un proceso de construcción de nuevos hoteles, pero también de cierre de establecimientos

Tabla 1. Variables evolutivas básicas de la actividad turística en Barcelona (1993-2013)

\begin{tabular}{|c|c|c|c|c|c|c|c|c|c|c|c|c|}
\hline Variables & 1993 & 1995 & 2000 & 2005 & 2006 & 2007 & 2008 & 2009 & 2010 & 2011 & 2012 & 2013 \\
\hline \multicolumn{13}{|l|}{ Hoteles } \\
\hline Total establ. & 155 & 160 & 187 & 268 & 285 & 295 & 310 & 321 & 328 & 339 & 352 & 36 \\
\hline 5 estrellas & 5 & 6 & 6 & 13 & 15 & 16 & 19 & 20 & 21 & 23 & 24 & \\
\hline 4 estrellas & 46 & 47 & 56 & 98 & 109 & 115 & 121 & 125 & 130 & 137 & 142 & 15 \\
\hline 3 estrellas & 60 & 60 & 70 & 93 & 97 & 98 & 104 & 107 & 111 & 113 & 119 & 11 \\
\hline estrellas & 18 & 21 & 28 & 34 & 34 & 35 & 36 & 36 & 34 & 34 & 34 & \\
\hline 1 estrella & 26 & 26 & 27 & 30 & 30 & 31 & 30 & 33 & 32 & 32 & 33 & \\
\hline Total plazas & 26.191 & 27.988 & 31.338 & 49.235 & 52.484 & 54.036 & 56.984 & 60.331 & 61.942 & 63.528 & 65.100 & 67.567 \\
\hline 5 estrellas & 2.136 & 2.989 & 3.027 & 7.200 & 7.832 & 7.954 & 8.487 & 8.853 & 9.083 & 9.309 & 8.723 & 9.193 \\
\hline 4 estrellas & 13.295 & 13.290 & 15.135 & 23.808 & 25.863 & 27.224 & 28.486 & 30.772 & 32.189 & 33.082 & 34.181 & 36.014 \\
\hline estrellas & 7.528 & 8.142 & 8.892 & 12.675 & 13.237 & 13.283 & 14.213 & 14.612 & 15.221 & 15.522 & 16.422 & 16.023 \\
\hline 2 estrellas & 1.817 & 2.132 & 2.788 & 3.667 & 3.667 & 3.650 & 3.828 & 3.845 & 3.354 & 3.475 & 3.523 & $3.94 \mathrm{C}$ \\
\hline 1 estrella & 1.415 & 1.435 & 1.496 & 1.885 & 1.885 & 1.925 & 2.970 & 2.249 & 2.095 & 2.140 & 2.251 & 2.388 \\
\hline \multicolumn{13}{|c|}{ Turismo de reuniones } \\
\hline Delegados & 175.323 & 167.593 & 269.508 & 343.005 & 491.028 & 629.704 & 690.908 & 576.157 & 616.833 & 647.693 & 597.610 & 583.956 \\
\hline Reuniones & 710 & 739 & 1.380 & 1.353 & 1.303 & 1.775 & 2.482 & 1.857 & 2.138 & 2.283 & 2.176 & 2.03 \\
\hline \multicolumn{13}{|l|}{ Cruceros } \\
\hline N. cruceros & 250 & 356 & 495 & 689 & 714 & 817 & 887 & 799 & 841 & 881 & 774 & 83 \\
\hline Pasajeros & 152.082 & 233.389 & 572.571 & 1.228 .561 & 1.407.179 & 1.768 .306 & 2.076 .585 & 2.151 .461 & 2.350 .283 & 2.657 .244 & 2.408 .634 & 2.599 .23 \\
\hline \multicolumn{13}{|l|}{ Bus turístico } \\
\hline Pasajeros & 101.400 & 131.600 & 873.611 & 1.654 .145 & 1.881 .125 & 2.181 .599 & 2.076 .581 & 1.913 .419 & 1.925 .226 & 2.125 .960 & 1.946 .907 & 1.985 .89 \\
\hline \multicolumn{13}{|l|}{ Aeropuerto } \\
\hline $\begin{array}{l}\text { Pasajeros Total } \\
\text { (mil) }\end{array}$ & $9.654,2$ & $11.727,8$ & $19.809,5$ & $27.152,7$ & $30.898,3$ & 32.898 .2 & $30.272,1$ & 27.421 .7 & $29.209,5$ & $34.398,2$ & $39,145,2$ & 35.210, \\
\hline $\begin{array}{l}\text { Pasajeros (mil) } \\
\text { internacionales }\end{array}$ & $3.948,6$ & $4.798,9$ & $9.524,4$ & $13.690,1$ & $15.555,4$ & $17.679,5$ & $17.691,6$ & $16.236,8$ & $17.574,7$ & $21.714,7$ & $23.679,3$ & 25.030 \\
\hline
\end{tabular}

Fuente: elaboración propia a partir de Turisme de Barcelona (diversos años). 
de categoría baja y de reformas y transformaciones que en muchos casos han supuesto un cambio de categoría para adaptarse a las nuevas demandas. El análisis de la evolución y el crecimiento en términos cuantitativos se complementa con la consideración de su dimensión cualitativa. En este sentido, y en cuanto a la composición de la oferta hotelera, se puede afirmar que en los dos últimos decenios se ha "cualificado" el censo hotelero: hoy más del $60 \%$ de las plazas son de cuatro y cinco estrellas, lo que contrasta con la situación de hace veinticinco años. Además, en la última década Barcelona ha doblado su oferta de hoteles de cinco estrellas y lujo, que ha pasado de 13 a 26. La mayoría de los proyectos se han ubicado en el Ensanche (Eixample), el centro histórico y el nuevo distrito 22@ (Poblenou). A lo largo de 2011 y 2012 se inauguraron 26 establecimientos, en su mayoría de cuatro estrellas.

El número de viajeros en establecimientos hoteleros y el volumen de pernoctaciones también han evolucionado de manera positiva, lo que avala el comportamiento expansivo del turismo en la ciudad. En 1990 el número de viajeros en establecimientos hoteleros fue de 1,7 millones con 3,8 millones de pernoctaciones; en el año 2000 se pasó a 3,1 millones de viajeros y 7,7 millones de pernoctaciones y, según los últimos datos disponibles, en el 2013 se alcanzó otro récord: 7,57 millones de viajeros con 16,48 millones de pernoctaciones.

Los datos sobre el comportamiento de la demanda continúan siendo uno de los retos en el conocimiento preciso de los componentes del sistema turístico pese a las aportaciones específicas sobre la segmentación de la demanda (André, 1999). Las estadísticas sobre el tema publicadas cada año por Turisme de Barcelona, a partir de su sistema de encuestas, constituyen la información más valiosa de que se dispone, de la que en esta ocasión se seleccionan algunos datos básicos.

En el año 2000 la estancia media de los viajeros fue de 2,5 días y, después de una disminución en el segundo quinquenio de la década (1,93 en 2005 y 1,97 en 2010), en los últimos cuatro años ha cambiado la tendencia hasta alcanzar los 2,18 días en 2013. El grado de ocupación de los establecimientos ha seguido igualmente una evolución favorable en los últimos veinticinco años, con algunas oscilaciones. En 2013 fue del 76,8\% en el caso de las habitaciones y del $67,5 \%$ en el de las plazas. Estos porcentajes pueden considerarse elevados si se tiene en cuenta el fuerte incremento de la capacidad de alojamiento hotelero de la ciudad (tanto hoteles como otros tipos de alojamiento) y la evolución histórica de este parámetro.

En relación con el origen de los turistas en hoteles hay que señalar un cambio estructural que se ha dado a lo largo de las dos últimas décadas: la proporción de visitantes españoles ha disminuido notablemente, y de ser mayoritaria a principios de los noventa, en 2005 representaba el 30\% del total del flujo y en 2013 tan solo el $20 \%$, por lo que el $80 \%$ del total son turistas internacionales. De los visitantes españoles, destacan los procedentes de Madrid (casi un tercio del conjunto español, sin contar los propios catalanes), mientras que del total del flujo de visitantes internacionales el $60 \%$ son europeos, proporción que se ha mantenido con tendencia a la baja, y principalmente son franceses, 
ingleses, italianos y alemanes. Del resto del mundo destaca la proporción de norteamericanos (en torno al $14 \%$ de este grupo), con un notable crecimiento a lo largo del periodo estudiado.

Barcelona capta una gama diversa de visitantes que se pueden simplificar en tres tipos básicos:

- Los viajeros turistas que pernoctan en la ciudad. Entre los viajeros que utilizan el alojamiento hotelero destaca, en primer lugar, el importante flujo de turismo por motivos "vacacionales", que supuso en 2013 casi el 51\%; y, en segundo lugar, aparece el flujo por motivos "profesionales» (negocios, ferias y congresos), que en 2013 representó el 41\%. El resto, un 8\%, lo constituye el flujo que engloba otros motivos. En la composición de los turistas según sus motivaciones se ha dado un cambio estructural desde 1997/1998: el turismo "profesional» fue superado por el turismo "vacacional», que logró alcanzar el $51,5 \%$, pero a partir del año siguiente empezó a perder valor relativo y en 2003 fue superado de nuevo por el turismo "profesional» solo coyunturalmente, ya que en los años siguientes de nuevo el turismo vacacional se situó primero, posición que mantiene hoy día.

- Los viajeros turistas que no pernoctan. Barcelona es frecuentada durante el día - a veces solo durante unas horas - por centenares y miles de turistas diarios que proceden de zonas turísticas más o menos próximas (el Maresme, la Costa Brava, la Costa Daurada) y que tienen la ciudad como un complemento o extensión de su destino principal.

- Los turistas que viajan en cruceros y que no pernoctan en los establecimientos de la ciudad, pero que durante la escala en Barcelona visitan la ciudad. Este flujo ha aumentado considerablemente en la última década y alcanzó el récord en 2011 con 2,65 millones y 2,59 en 2013 (Tabla 1). Cada vez más, una buena parte de cruceristas también pernocta en la ciudad al convertirse el puerto en origen y final de diversos cruceros, lo que comporta en muchas ocasiones dormir en la ciudad antes de la salida y después de la llegada.

La oferta de alojamiento de la ciudad se ha ampliado de manera notable en los últimos años con la aparición de otros tipos de establecimientos, que se suman a los hoteles, y ha posibilitado un incremento destacado de la capacidad de alojamiento de la ciudad. Su dimensión no es fácil de determinar dada la naturaleza de la oferta y los mecanismos de registro existentes. En los últimos cinco años Barcelona ha vivido una eclosión de los denominados "pisos turísticos» — buena parte de los cuales funcionan sin la correspondiente licencia- y del alquiler de habitaciones para turistas, modalidades que basan su éxito en buena medida en su comercialización por internet. La oferta de alojamiento total en 2013, en plazas y según datos de Turisme de Barcelona (2014), es la siguiente: 67.567 plazas en hoteles; 28.176 plazas en viviendas de uso turístico; 6.782 plazas en albergues y 5.683 plazas en pensiones y hostales. En conjunto, más de 110.000 plazas.

La dimensión de pernoctaciones en establecimientos hoteleros y similares representa, según estimación de Turisme de Barcelona (2014), el 2\% del con- 
junto de pernoctaciones que se realizan en Barcelona por parte de la población residente permanente y la no permanente. Las estimaciones indican además que, si se consideraran todas las pernoctaciones de los no residentes (establecimientos no regulados, familiares, etc.), la cifra alcanzaría el 3,6\% del total. La dimensión del flujo turístico se incrementa notablemente si se suma a las pernoctaciones el movimiento diario de turistas procedentes de otros municipios y zonas turísticas cercanas, es decir el flujo de "excursionistas», según la conceptualización institucional. El cálculo de estos desplazamientos presenta dificultades y, según las estimaciones realizadas, los visitantes de Barcelona (incluyendo turistas y excursionistas, es decir, no alojados) fue de 24,5 millones en 2007 y de 27,1 millones en 2012.

\section{Distribución espacial de la actividad turística: los cambios en el mapa turístico}

Las aproximaciones sobre la relación turismo/ciudad se realizan a una escala que considera habitualmente el conjunto de la ciudad. En este caso, Barcelona como un espacio único y homogéneo, asimilado al municipio en términos operativos y estadísticos. Interesa, sin embargo, considerar la localización del turismo en el plano de la ciudad, su distribución por barrios, sus pautas espaciales que permiten constatar la existencia de zonas turísticas y zonas no turísticas y de un mayor o menor grado de concentración del turismo y una mayor o menor afectación en los distintos barrios de la ciudad.

El mapa actual de localización hotelera de la ciudad presenta unas pautas diferentes, en buena parte, al mapa de hace veinticinco años, cuando la hotelería aparecía concentrada casi exclusivamente en el centro de la ciudad. Sin embargo, hoy se encuentran hoteles en todos los distritos y en buena parte de barrios, si bien con intensidades diferentes, lo que dibuja un "mapa turístico» asimétrico conformado, a su vez, por la desigual localización de los recursos y productos turísticos y por las pautas espaciales de la movilidad de los turistas. Se trata de un proceso general de difusión espacial del turismo por toda la ciudad, si bien los distritos centrales continúan manteniendo el principal protagonismo.

El análisis de la evolución del equipamiento hotelero (hoteles, hotelesapartamentos y pensiones y hostales) de Barcelona por distrito, en los últimos diez años (ver los datos concretos en la Tabla 2), permite identificar hasta cuatro procesos específicos: 1) densificación del centro histórico (Ciutat Vella); 2) difusión intensa hacia el Ensanche (centro de la ciudad); 3) difusión intensa hacia el distrito de Sant Martí; y 4) difusión débil hacia el resto de los distritos, menos céntricos.

La reorganización del espacio turístico también ha modificado los lugares que hay que visitar, los «iconos» de la trama urbana (atributos del territorio que deben ser conocidos), aunque solo parcialmente, ya que los cambios de este proceso son más lentos al situarse en el ámbito de la imagen y del imaginario social. En 2013, el templo de la Sagrada Familia fue con diferencia el más visitado, y lo 
Tabla 2. Evolución del equipamiento hotelero de Barcelona por distritos (2004-2013)

\begin{tabular}{|c|c|c|c|c|c|c|}
\hline \multirow[b]{2}{*}{ Distritos } & \multicolumn{2}{|c|}{2004} & \multicolumn{2}{|c|}{2008} & \multicolumn{2}{|c|}{2013} \\
\hline & Establ. & Plazas & Establ. & Plazas & Establ. & Plazas \\
\hline 1. Ciutat Vella & 184 & 12.406 & 187 & 14.746 & 213 & 20.114 \\
\hline 2. Eixample & 112 & 9.132 & 149 & 13.937 & 212 & 17.982 \\
\hline 3. Sants-Montjuïc & 29 & 4.965 & 34 & 5.686 & 42 & 7.161 \\
\hline 4. Les Corts & 20 & 5.292 & 21 & 5.885 & 22 & 6.180 \\
\hline 5. Sarrià-Sant Gervasi & 38 & 3.629 & 41 & 3.567 & 44 & 3.751 \\
\hline 6. Gràcia & 11 & 467 & 14 & 481 & 18 & 935 \\
\hline 7. Horta-Guinardó & 8 & 526 & 10 & 999 & 12 & 1.023 \\
\hline 8. Nou Barris & 3 & 349 & 2 & 316 & 2 & 316 \\
\hline 9. Sant Andreu & - & - & 1 & 180 & 2 & 222 \\
\hline 10. Sant Martí & 11 & 1.505 & 21 & 6.563 & 36 & 11.444 \\
\hline Total & 417 & 38.583 & 480 & 52.360 & 603 & 69.128 \\
\hline
\end{tabular}

Fuente: elaboración propia a partir de Estadístiques Ajuntament de Barcelona.

Tabla 3. Lugares de interés más visitados en Barcelona (1994-2013)

\begin{tabular}{|c|c|c|c|c|c|c|c|c|}
\hline * & Lugar de interés & 1994 & 2000 & 2005 & 2010 & 2011 & 2012 & 2013 \\
\hline 1 & Templo de la Sagrada Familia & 700.000 & 1.420 .087 & 2.376 .780 & 2.317 .349 & 3.202 .000 & 3.233 .526 & 3.176 .970 \\
\hline 2 & L'Aquàrium & ** & 1.563 .493 & 1.346 .912 & 1.514 .570 & 1.675 .735 & 1.647 .163 & 1.718 .380 \\
\hline 3 & Museo del FC Barcelona & 538.077 & 1.156 .090 & 1.133 .733 & 1.311 .638 & 1.626 .990 & 1.540 .648 & 1.506 .022 \\
\hline 4 & Pueblo Español & 1.421 .396 & 1.478 .546 & 1.305 .557 & 936.455 & 1.323 .578 & 1.223 .875 & 1.258 .645 \\
\hline 5 & Castillo de Montjuïc & ** & ** & ** & 1.371 .355 & 1.318 .000 & 1.159 .042 & 1.072 .000 \\
\hline 6 & Parque Zoológico & 1.025 .172 & 930.000 & 963.146 & 1.054 .885 & 1.164 .027 & 1.080 .187 & 1.070 .104 \\
\hline 7 & $\begin{array}{l}\text { La Pedrera. } \\
\text { Fundació Caixa Catalunya }\end{array}$ & ** & 1.386 .721 & 1.229 .496 & 973.603 & 962.293 & & 944.509 \\
\hline 8 & Museo Picasso & 711.103 & 1.026 .549 & 1.074 .060 & 1.065 .388 & 1.057 .399 & 948.869 & 915.226 \\
\hline 9 & CosmoCaixa Barcelona & 419.709 & 262.561 & 1.385 .116 & 721.337 & 830.282 & 788.176 & 716.877 \\
\hline 10 & CaixaFòrum Barcelona & 162.821 & 344.097 & 967.110 & 1.241 .572 & 782.529 & 971.101 & 686.151 \\
\hline
\end{tabular}

Fuente: elaboración propia a partir de Turisme de Barcelona.

* Ordenados según volumen de visitas de 2013

${ }^{* *}$ Sin datos

sigue siendo desde que en 2001 alcanzó la primera posición de lugares de interés de la ciudad más frecuentados (Tabla 3). El resto de lugares más visitados de Barcelona lo conforman tanto equipamientos lúdicos como culturales y museos. La evolución muestra que en los años noventa los equipamientos más frecuentados eran los de carácter lúdico, y progresivamente han irrumpido con fuerza los culturales y museos, si bien en los últimos años se observa un estancamiento en su frecuentación o incluso un descenso de las cifras.

En relación con la puesta en valor turístico de nuevos lugares de la ciudad, con la creación de nuevos productos y con la nueva movilidad del flujo 
turístico, se deben mencionar de forma destacada, por una parte, el papel del Barcelona Bus Turístico (producto turístico creado en 1988 y gestionado posteriormente por Turisme de Barcelona) (ver los datos en la Tabla 1) y, por otra parte, la implementación de las acciones del Plan Estratégico de Turismo de la Ciudad de Barcelona 2015 (en el apartado 6 se explican sus características), orientadas a la "desconcentración del turismo", con la redacción y el desarrollo de los Planes de Turismo de Distrito.

Los Planes de Turismo de Distrito de Barcelona son instrumentos previstos en el Plan 2015, en concreto en el reto sobre «Desconcentración de la actividad turística», y con ellos se persigue un doble objetivo: poner en valor los recursos turísticos de cada distrito y conseguir descentralizar y descongestionar el centro de la ciudad. Los planes, que prevén en cada caso la identificación de estrategias y un plan operativo, son promovidos y dotados económicamente por el gobierno municipal, y su redacción e implementación se inició en 2012. Afectará a siete distritos, el primero de los cuales ha sido el de Nou Barris, considerado uno de los distritos con más potencial de desarrollo.

Desde una aproximación espacial y como síntesis del proceso de conversión de Barcelona en ciudad turística, se distinguen dos espacios turísticos diferenciados:

- El espacio turístico tradicional. Configurado durante una etapa anterior e intensificado en el último periodo. Aparece dibujado sobre todo en el centro histórico y el Ensanche (sucesión de iconos entre la Rambla, el paseo de Gràcia y la Sagrada Família).

- El nuevo espacio turístico. Se superpone sobre el espacio tradicional, lo integra y lo desborda como consecuencia de una extensión espacial del turismo en otras partes de la ciudad, no de manera continua físicamente, por lo que se identifican unas piezas discontinuas, fragmentadas. El nuevo espacio ha incorporado nuevas piezas urbanas y la estructura resultante es polinuclear. Se caracteriza por presentar una situación intermedia entre una distribución concentrada y una distribución dispersa. Comprende nuevos atributos de la geografía turística de la ciudad: la zona del Fòrum y Diagonal Mar; el Port Olímpic/Vila Olímpica; el Frente Portuario (Barceloneta, Port Vell, Maremagnum, World Trade Center) y la montaña de Montjuïc y Diagonal montaña (FC Barcelona, eje comercial y Pedralbes). En los últimos cinco años se suman las nuevas zonas de centralidad turística, en proceso de configuración y de amplitud y especialización diferenciadas: la plaza de España-estación de Sants; el Paral.lel; la rambla del Poblenou y entorno y la plaza de Les Glòries y su entorno («nueva plaza turística»).

El desarrollo turístico inducido por la ciudad en los últimos años ha provocado, además de un nuevo mapa turístico de Barcelona, un cambio de su escala espacial. El espacio turístico real ha sobrepasado la ciudad en sentido estricto, se ha extendido más allá del municipio de Barcelona y se ha configurado un espacio turístico funcional de límites imprecisos. Tal como se ha mencionado 
en el apartado anterior, conceptualmente se utiliza el término «destino Barcelona" para referirse a este espacio turístico real. El análisis y la explicación del paso de una Barcelona "ciudad turística» a la nueva realidad que supone el «destino Barcelona» constituyen un reto que desborda los objetivos de esta investigación.

\section{Articulación del turismo en las estructuras preexistentes: afectaciones del turismo en la ciudad}

La realidad del turismo en Barcelona es compleja — cada vez más_ y su articulación en las estructuras preexistentes (formales o urbanísticas y funcionales de base social y económica) no está exenta de problemas y conflictos de diversa naturaleza. El escenario actual de relación entre turismo y ciudad se manifiesta en afectaciones positivas y en afectaciones negativas, y se proyecta sobre todos los ámbitos (espacio público, tejido empresarial, ciudadanía, instituciones, etc.). Esta afirmación se puede corroborar con datos parciales o sectoriales que se disponen sobre los efectos del turismo en la ciudad y con las aportaciones que sobre el tema se han llevado a cabo. En los últimos cinco años dichas aportaciones (estudios, debates y reflexiones sobre turismo en la ciudad) se han multiplicado debido, por una parte, a la movilización que supuso la redacción entre 2008 y 2010 del Plan Estratégico de Turismo de la Ciudad de Barcelona 2015 (proceso participativo y documentos del plan) y, por otra parte, a las respuestas (ciudadana, académica, institucional, mediática, etc.) a los efectos de la intensificación del turismo y su mayor percepción como conflicto en el estadio actual de mayor desarrollo de las actividades turísticas y de formalización de la relación turismo-ciudad.

A partir del conocimiento formalizado y de las informaciones difundidas a través de los medios de comunicación, se identifican como aproximación global y sistematizada cuatro ámbitos donde se pueden observar las afectaciones del turismo:

a) La estructura de producción y consumo, y en particular el impacto económico del turismo.

b) El uso de la ciudad, los conflictos por el uso de los espacios públicos (y privados) y la relación entre residentes y visitantes.

c) La imagen de la ciudad, la marca Barcelona, en un escenario de globalización y de competencia entre ciudades.

d) Los discursos sobre los significados del turismo y su gestión pública. Este ámbito será abordado en el siguiente apartado.

\subsection{El impacto económico del turismo}

La complejidad de la estimación de los impactos turísticos convierte el interés por medir este tipo de afectación en un reto. Según los resultados para 2007 (AQR-IREA, 2009), la actividad turística en Barcelona generaba entre die- 
ciocho y veintidós millones de euros al día, con lo que el impacto en cifras absolutas oscilaría entre los seis mil quinientos y los ocho mil millones de euros anuales, lo que supone aproximadamente entre un 8 y un 10\% del PIB de la ciudad.

El impacto se distribuye entre un amplio abanico de subsectores económicos y cabe destacar que aproximadamente dos terceras partes del impacto total se generan por los alojados en establecimientos hoteleros. También cabe destacar el casi $10 \%$ que generan los visitantes de día o excursionistas. Por otra parte, se observa un importante efecto multiplicador de la actividad turística en una ciudad como Barcelona. Según AQR-IREA (2009), si se consideran los efectos indirectos del turismo (aquellos que se registran en el resto de sectores de la economía fruto de la demanda de bienes y servicios realizada por los sectores más "turísticos» para hacer frente a la demanda generada por los visitantes), estos oscilan entre un 20 y un $30 \%$ del total según los diferentes escenarios metodológicos planteados, lo que supone una cifra que se sitúa entre los 1.300 y los 2.600 millones de euros anuales. Estos datos ilustran el gran efecto multiplicador de la actividad turística, que implica a sectores tan diversos como el textil, el inmobiliario o el financiero, entre muchos otros.

Las últimas estimaciones sobre el tema y referidas a 2013, según Turisme de Barcelona (2014), muestran que el impacto económico directo del turismo es de 27,7 millones de euros al día, cifra superior a la de 2007, que se situó entre 18 y 22 millones de euros al día. En relación con la población ocupada, en 2013 trabajaban en el sector más de 120.000 personas, que superaron las 100.000 estimadas en 2007.

\subsection{Usos del espacio público y dinámicas socioeconómicas}

Las primeras aproximaciones sobre las afectaciones de carácter espacial del turismo conducen a constatar que los conflictos asociados al hecho turístico no son inducidos exclusivamente por el turismo, sino que el turismo es uno más entre los diversos factores de la génesis de dichos conflictos y del cambio global que se desarrolla en las ciudades. Barcelona está experimentando una presión turística y recreativa, fruto de la masificación del turismo, con unos efectos de concentración y congestión que pueden ser no deseables para los residentes o insatisfactorios para los turistas, y con unas situaciones de fricción entre operadores del sector. Los analistas abordan esta problemática interpretándola como conflictos inherentes a los propios procesos de producción-consumo turísticos en un escenario urbano.

Nunca antes de los últimos cinco años se había puesto de manifiesto de forma tan explícita y organizada la respuesta de la ciudadanía, intensificada en 2014 y 2015, sobre estos temas, que trasciende el ámbito de lo particular asociado a casos concretos y que se generaliza y crea estado de opinión crítica respecto al «modelo turístico actual». Esta respuesta y movilización están obligando al gobierno municipal a reaccionar para solucionar los problemas, mitigar los efectos no deseados o buscar alternativas. El equipo de gobierno, 
desbordado ante los acontecimientos y la dimensión del debate sobre un sector turístico que es clave y con problemas de encaje en la ciudad, decidió a principios de 2015 dos acciones: celebrar tres encuentros multisectoriales, en tres días, antes de los comicios locales de mayo de 2015; y, en segundo lugar, celebrar a finales de febrero una insólita audiencia pública para discutir el modelo turístico de Barcelona.

Las asociaciones de vecinos presentaron, a través de la Federación de Asociaciones de Vecinos de Barcelona (FAVB), sus propuestas en la audiencia pública celebrada el 24 de febrero de 2015. Su decálogo de propuestas ilustra perfectamente la naturaleza y el alcance de los conflictos, tal como se deduce de su relación:

1) Destinar la tasa turística a la ciudad (no solo a la promoción).

2) Moratoria sobre la concesión de nuevos hoteles (previo establecimiento con los vecinos de los límites de plazas por distrito).

3) Considerar los pisos turísticos bajo el régimen administrativo de los hoteles y no como vivienda.

4) Congelación total de licencias de pisos turísticos (hasta que no se cumpla el punto anterior).

5) Regulación fiscal del alquiler de habitaciones (incluyendo la tasa turística).

6) Plan especial para proteger el comercio y defensa del comercio de proximidad.

7) Diseños urbanísticos más participativos.

8) Definir el aforo de las zonas monumentales más frecuentadas y la ocupación del espacio público (con revisión de ordenanzas sobre terrazas en la vía pública).

9) Nuevas medidas de protección ambiental (relación con el tráfico).

10) Crear trabajo de calidad.

En relación con el primer punto de este decálogo, cabe añadir que la tasa turística es una figura impositiva de Cataluña, aplicable a las estancias turísticas, que entró en vigor en noviembre de 2012, después de años de debates y polémicas sobre su implantación. La tasa turística catalana recaudó 41 millones de euros en 2014, más de la mitad de los cuales (el 52\%) corresponden a Barcelona y municipios limítrofes. La ley prevé que un 30\% de lo recaudado lo gestionen los municipios turísticos en cada caso. Hoy el debate en Barcelona se sitúa en reivindicar una mayor proporción y, por otro lado, orientar su aplicación no solo hacia inversiones en promoción sino también a paliar los efectos negativos del turismo en la ciudad y, en particular, en las zonas más afectadas por los conflictos.

La situación de los espacios congestionados por el turismo, que han alcanzado una alta especialización turística, da paso a hablar de tematización turística y de la conversión de determinadas zonas en "parques temáticos» (la Rambla, el Born, el Raval...). Agregando las zonas indicadas a otras contiguas se percibe todo el centro histórico de la ciudad como un gran parque temático para el 
turismo. El turismo no afecta igual a todos los barrios y las zonas que registran más conflictos (tal como recogen los medios de comunicación) son el Park Güell, la Sagrada Família, Gràcia, la Barceloneta, Ciutat Vella y el Poble Sec, principalmente.

\subsection{La imagen de la ciudad, la marca Barcelona y Barcelona como «marca»}

La competencia entre ciudades es cada vez mayor y su imagen ante el mundo globalizado se ha convertido en un activo estratégico para sus ciudadanos por el valor relacional, social, económico y cultural de la marca. Por ello, la llamada «marca Barcelona» es un valor añadido de competitividad para la exportación, la captación de compradores y visitantes y la atracción de inversiones y talento.

La marca Barcelona tiene una gran potencia internacional y se asocia al éxito, a la innovación, al talento, a la creatividad y a una posición de vanguardia. Y es que la Ciudad Condal, cuya fama internacional no para de crecer, destaca en campos como el turismo, la cultura, la arquitectura, la gastronomía, el diseño, la moda, la salud, la investigación, la educación y las nuevas tecnologías; pero también es un referente para la industria automovilística, la farmacéutica o la alimentaria. Barcelona es una ciudad donde apetece trabajar y vivir, tal como revelan las clasificaciones de ciudades que sobre este tema publican diversas entidades e instituciones internacionales.

Barcelona se ha consolidado internacionalmente como capital del turismo cultural y se perfila como centro de innovación, pero ha de fortalecer una imagen propia y diferenciada como ciudad de negocios. La creación de la marca Barcelona y su proyección tiene que ver con el modelo de ciudad construido y con la promoción del denominado modelo Barcelona (García García, 2013).

La potencia y la visibilidad del conjunto de Barcelona como marca y los esfuerzos para su creación e intensificación, su asociación a un estatus de éxito, contrasta con la realidad de la ciudad observada a otras escalas, de la ciudad dual, fruto del incremento en los últimos años de las desigualdades sociales y los desequilibrios entre barrios. Una realidad cotidiana conformada también por las manifestaciones de conflicto asociadas al turismo. La vinculación del modelo de ciudad a la «marca» es vista como una disfunción y se reivindica la Barcelona ciudad frente a la Barcelona marca. La conferencia impartida por Marina Garcés (2014), el 23 de mayo de 2014 en el Centro de Cultura Contemporánea de Barcelona (CCCB), titulada «Desmarcar Barcelona», supone una destacada referencia de las miradas críticas sobre los desafíos que plantea el turismo de masas y sobre la relación entre turismo y espacio público. La visión crítica, no obstante, es anterior a los últimos años de intensificación del turismo y de crisis económica, y surge con fuerza en plena expansión económica de la primera década del siglo XXI relacionada con la propia dinámica urbana y la gestión pública del denominado «modelo Barcelona» (Capel, 2005; Delgado, 2007). 


\section{Actores del proceso y protagonismo de la gestión pública del turismo}

El análisis del proceso de desarrollo del turismo en Barcelona y la conversión de la capital de Cataluña en ciudad turística permiten identificar el papel de diversos actores. En la bibliografía sobre el tema aparece destacado de manera recurrente el protagonismo que ha tenido la gestión pública del turismo (López Palomeque, 2002). En particular, en el proceso de desarrollo de las actividades turísticas han sido clave las estrategias para el fomento y la promoción llevadas a cabo por los entes de promoción. Destaca en este proceso el papel del consorcio Turisme de Barcelona, creado en 1993. El origen del ente Turisme de Barcelona debe buscarse, de hecho, en la estrecha colaboración iniciada en 1989 entre el Patronato de Turismo del Ayuntamiento y la Cámara Oficial de Comercio, Industria y Navegación para la realización del Plan de Marketing Turístico de Barcelona previamente a los Juegos Olímpicos de 1992.

La referencia a los Juegos Olímpicos de 1992 y los procesos asociados es ineludible tanto si se quiere tratar el sistema turístico de la ciudad como si se quiere indagar en las pautas de la reestructuración urbana y del modelo de ciudad. De hecho, los Juegos Olímpicos de 1992, por su condición de gran intervención urbana, son interpretados como pretexto y estrategia de reestructuración y crecimiento de la ciudad. Es probablemente el paradigma de la estrategia de la organización de megaeventos para dinamizar y construir la ciudad, que se remonta al siglo xIx. También se pretendió conseguir lo mismo con otro gran evento: el Foro de las Culturas 2004. Sin embargo, en la primera década del siglo ya se concibe todo el turismo (más allá de los megaeventos) como una realidad estratégica para la ciudad (Duran, 2004).

La puesta en marcha de Turisme de Barcelona significó, entre otros avances, la unificación de la actuación pública y privada en el ámbito del turismo, la inserción del turismo en el conjunto de la estrategia y de las prioridades de actuación de la ciudad recogidas en los diversos planes estratégicos de la ciudad y del ámbito metropolitano, y la centralización en un único organismo de las acciones de promoción turística de la ciudad y, en particular, de la coordinación de actividades y eventos (Delàs, 1995).

El modelo organizativo y la filosofía de actuación en materia de turismo en la ciudad de Barcelona han evolucionado. La formalización más evidente de estos cambios y del avance en la gestión de la relación ciudad-turismo, más allá de la labor de promoción, la constituye la aprobación en julio de 2008 de la realización del Plan Estratégico de Turismo de la Ciudad de Barcelona 2015. Este nuevo instrumento de gestión fue promovido por el Ayuntamiento y encargado a Turisme de Barcelona, con la pretensión de afrontar una nueva etapa caracterizada por la finalización del crecimiento acelerado en el sector, garantizar el posicionamiento de Barcelona como destino turístico mundial y buscar una mejor conciliación del turismo en la ciudad. El plan tiene su origen en el Plan de Acción Municipal 2008-2011 (PAM), que preveía la Línea de actuación 3.8 dedicada a Turismo. Entre sus acciones concretas figura la elaboración de un plan estratégico de turismo de la ciudad con el fin de iniciar 
un proceso de reflexión sobre el modelo de turismo en la ciudad, definir las futuras estrategias de actuación y establecer actuaciones.

El Plan 2015 se redactó entre 2008 y 2010 (Ajuntament de Barcelona, 2010). Su implementación se inició con un ritmo lento, marcado por la proximidad y la incertidumbre de las elecciones municipales que tenían que celebrarse en menos de un año. Los comicios locales de mayo de 2011 supusieron un cambio de partido político en el gobierno de la ciudad. Por primera vez, desde las primeras elecciones municipales de 1979, un partido conservador ( $\mathrm{CiU})$ gobernaba la ciudad, sin mayoría absoluta y sin pactos estables de gobierno. En este contexto político-institucional, el nuevo equipo de gobierno de la ciudad (2011-2015) incorporó el Plan 2015 como uno de los instrumentos para la gestión del turismo. Por su parte, el ente Turisme de Barcelona (consorcio público-privado participado por el Ayuntamiento de Barcelona, la Cámara de Comercio, Industria y Navegación de Barcelona y la Fundación Barcelona Promoción) desarrolla sus estrategias y acciones en pro del fomento y promoción turística de la ciudad.

El Plan Estratégico de Turismo de la Ciudad de Barcelona 2015 pretendía conformar una orientación estratégica por parte de los diversos agentes turísticos de la ciudad, con dos objetivos concretos: mejorar el encaje entre el turismo y la ciudad y mejorar la competitividad de la actividad turística de Barcelona (Serra, 2010). Además, cabe subrayar como rasgos destacados el período dedicado a su redacción (dos años no es tiempo habitual), su concepción (turismo y ciudad, más allá de un plan de promoción o fomento del turismo) y su metodología innovadora. En conjunto, constituyó un proceso sin precedentes en la redacción de planes estratégicos e instrumentos de gestión.

Cinco años después de ser aprobado, es posible hacer un primer balance provisional de la implementación del Plan Estratégico de Turismo de la Ciudad de Barcelona 2015. En esta ocasión se aportan algunos datos y valoraciones básicas. El Plan 2015 prevé una propuesta de actuaciones con diez programas y un total de ciento cincuenta acciones. El seguimiento del grado de ejecución permite afirmar que su implementación se ha realizado de manera muy diferente en función del ámbito en el que trabajar y de la entidad promotora encargada de su puesta en marcha. A modo de resumen, las acciones realizadas suponen el $18 \%$; las que todavía se están llevando a cabo, el 56\%; y el conjunto que no se está trabajando, un 26\% del total (Bonilla, 2014: 77).

Hasta 2010, según Palou (2010: 772), en Barcelona no existía una integración ni adaptación de la actividad turística en los órganos de gestión y administración municipales, hecho que tiene que ver en parte con la creencia consentida durante años de que el turismo era, fundamentalmente, un «sector económico» que causaba un impacto económico positivo en la sociedad, pero sobre todo en el mundo de los servicios directamente vinculados con esta actividad.

Del seguimiento de las acciones realizadas en la implementación se identifican ocho que se consideran más significativas: la gobernanza turística; el fortalecimiento de la Mesa Técnica Municipal (MTM); la desconcentración 
territorial, con los Planes de Turismo de Distrito; la inteligencia turística; el turismo responsable y sostenible; las nuevas medidas de gestión, regulación y control turístico; la nueva señalización turística y, finalmente, la generación de complicidades entre las administraciones (Bonilla, 2014: 81).

En relación con la gobernanza, en 2010 se creó el Departamento de Turismo y Eventos, integrado en la organización de Barcelona Activa (entidad encargada de ejecutar las políticas de promoción económica del Ayuntamiento de Barcelona), con la misión de impulsar las acciones relacionadas con la línea de la nueva gobernanza turística de la ciudad, incluyendo el despliegue operacional del Plan 2015. La creación de este departamento supuso dar una nueva visión de la actividad turística y asumir administrativamente su relevancia. Por primera vez en la historia, hay un área en el Ayuntamiento que se ocupa de gestionar la actividad turística de Barcelona. Según Suñol (citado por Bonilla, 2014: 82), la gran importancia del nuevo departamento radica en que una de las cosas que se han alcanzado plenamente con el Plan 2015 es que el turismo es una actividad absolutamente transversal dentro de la actuación municipal.

La Mesa de Turismo Municipal se creó en el año 2009 — antes de la aprobación del Plan 2015 - y se configuró como instrumento de coordinación transversal, con facultad de generar encargos a la organización municipal y vinculada a los órganos de gobierno de la administración municipal ejecutiva. En los últimos años se ha consolidado y reforzado su funcionamiento con dos medidas: ha elevado su nivel de representación política y ha creado en su seno la ponencia técnica de turismo y ciudad, un órgano convocado cada dos meses por la gerencia adjunta de proyectos estratégicos, encargado de impulsar las políticas transversales en turismo y con representación en todas las áreas.

Hoy el turismo en Barcelona sí afecta o forma parte de sus componentes morfológico o constructivo, funcional o relacional (uso económico o social) y simbólico e institucional. Más allá de las respuestas puntuales a los problemas generados por el turismo, hoy sí debería existir una concepción clara del turismo en el modelo de ciudad y la consecuente integración del turismo en el organigrama de gestión municipal, en la gestión de la ciudad (turística). ¿Es suficiente el nivel político administrativo que se ha alcanzado a través del Departamento de Turismo y Eventos y de la Mesa Técnica de Turismo? El debate sobre el turismo en Barcelona, sobre el modelo turístico de la ciudad, se ha incorporado a la agenda de los grupos políticos, en cuyos programas para las elecciones municipales de mayo de 2015 el turismo aparecerá como componente importante.

En relación con el rol del conjunto de los actores, hay que considerar que el proceso de conversión de Barcelona en ciudad turística no ha sido solo resultado de la acción de los actores locales, y que, en consecuencia, la realidad turística de la ciudad, su modelo turístico, es el resultado también de la participación y de los intereses de los actores externos (operadores turísticos y turistas), con lógicas e intereses que no siempre coinciden con las lógicas de los actores locales. Esta multiplicidad de actores ha de tenerse en cuenta para 
entender hoy la compleja realidad turística de la ciudad y también el dibujo del modelo turístico deseable.

El rol de estos actores aparece reforzado en los tiempos de la globalización (escenario internacional para los inversores) y de las continuas revoluciones en el orden tecnológico y de la comunicación, que posibilitan nuevos comportamientos del turista, nuevos negocios, nuevas relaciones de producción-consumo turísticas diferentes a las preexistentes, a veces fuera del ámbito de proyección de la Administración pública, de la gestión turística, de la gestión de la ciudad. $\mathrm{O}$ al menos requieren nuevas formas y mecanismos de gestión, de relación entre actores. Como muestras de estas afirmaciones, se puede mencionar el papel de las plataformas de opinión de los usuarios, con la creación de opinión sobre el destino Barcelona y sobre productos específicos, o bien los conflictos en torno a los nuevos negocios a través de internet de alquiler de coches (por ejemplo, Uber) o de alojamientos únicos de anfitriones (pisos, habitaciones) (por ejemplo, Airbnb).

\section{A modo de resumen y conclusiones}

En la evolución del turismo de Barcelona se pueden distinguir diversas etapas. A partir de una simplificación de la temporalización de la rica historia del turismo en la ciudad, se centra la atención en la última etapa, que corresponde a los últimos veinticinco años. Lo sucedido en este tiempo ha permitido que Barcelona pase de ser una ciudad con turismo a convertirse en una ciudad turística, tal como reflejan las evidencias aportadas.

Durante los últimos veinticinco años, la ciudad ha evolucionado desde un estadio caracterizado por la existencia de un sistema turístico bien organizado y eficiente a un estadio en el que Barcelona se ha convertido en ciudad turística. El proceso reciente de fuerte crecimiento de las actividades turísticas en la ciudad ha conducido a que el turismo haya alcanzado un carácter estructural, que se manifiesta tanto en sus componentes formales como en sus funciones urbanas. Y, obviamente, en los procesos de gestión de la ciudad, dado que el turismo afecta y forma parte de las relaciones y las realidades social, económica, política y espacial.

¿Qué explica el proceso de conversión de Barcelona en ciudad turística? Tal como se ha señalado, un conjunto de factores interrelacionados que se identifican y separan a efectos analíticos. Entre estos factores destaca el papel decidido de la Administración pública en favor del turismo a través de distintas instancias e instrumentos y el dinamismo de los actores locales y de los actores externos (los operadores turísticos de diversa naturaleza y los turistas).

La interpretación sistematizada en los apartados anteriores, en los que se han aportado evidencias sobre la intensificación del turismo en la ciudad y sobre las premisas iniciales en torno al destacado papel de la gestión pública en el proceso y sus nuevos retos, permite llegar a detectar determinados rasgos a modo de conclusión, reflexión y debate. Estas valoraciones se agrupan de forma selectiva en torno a cuatro ámbitos. 


\section{a) Barcelona, ciudad turistica y destino de primer orden internacional}

Barcelona se ha convertido en una ciudad turística que, además, se sitúa en un lugar destacado de la clasificación internacional y en particular en el mapa del turismo europeo. El turismo ha reorientado la imagen de la ciudad potenciando su percepción y su rango internacional a partir de la función turística. En relación con el desarrollo del turismo, su articulación en las estructuras preexistentes y sus afectaciones, se identifican entre otros los siguientes cambios:

1) $\mathrm{Ha}$ trascendido el sector turístico tradicional, que afecta a gran parte del tejido empresarial. Ha dinamizado la actividad económica de la ciudad y el impacto económico se ha incrementado de manera notable en los últimos diez años.

2) Ha ampliado el mapa turístico. Se observa un proceso de difusión del turismo por la mayor parte de los distritos (localización de los alojamientos, lugares visitados, etc.).

3) Ha ocupado (apropiado o invadido, según los analistas) unos espacios centrales (públicos y privados) hasta llegar en algunos casos a caracterizarlos (espacios tematizados) o a crear situaciones de conflicto con los usos y funciones preexistentes.

4) Ha provocado, por su intensidad y alcance, que el turismo se incorpore como componente clave del modelo de ciudad.

La dualidad detectada en la relación turismo-ciudad está en el centro del debate, que como afirma Oppenheimer (2015) es la doble vida de Barcelona: por una parte es admirada fuera y por otra parte los barceloneses se preguntan hacia dónde va la capital de Cataluña, con defensores y detractores del turismo.

\section{b) Gestión del turismo, gestión de la ciudad}

En las conclusiones del artículo «La estrategia del turismo metropolitano: el caso de Barcelona» (López Palomeque, 1995) se valoraban las estrategias para el desarrollo del turismo en Barcelona, entre las que destacaban la reorganización de los entes con responsabilidad en la promoción y fomento del turismo, con la creación de Turisme de Barcelona en 1993. También se mencionaba que el turismo "se tenía que contemplar en sí mismo como estrategia para el desarrollo de Barcelona, de su modelo de ciudad futura». Veinte años después, el futuro ya ha llegado: Barcelona es una ciudad turística y el turismo forma parte del modelo de ciudad y ello requiere que forme parte de su modelo de gestión, más allá de la existencia de una eficaz gestión de la promoción del turismo.

Abordar hoy día la gestión del turismo ya no supone considerar únicamente la gestión en torno a la promoción y el fomento de las actividades turísticas, sino que al constituir el turismo un componente estructural del modelo de ciudad se crea una nueva situación en la que el turismo se integra e impregna la gestión municipal, la gestión de la ciudad, no solo del sector. Hasta ahora, el modelo de gestión de la ciudad no se ha adaptado a la nueva función de la 
ciudad, a la función turística (Palou, 2010; Palou, 2012; Bonilla, 2014), si bien la creación de la Mesa Técnica de Turismo y el desarrollo de algunas acciones del programa Plan 2015 suponen un avance hacia la gobernanza de la ciudad turística. El tema es un reto inaplazable y muy probablemente en la próxima legislatura (a partir de mayo de 2015, tras las elecciones municipales) se tomen decisiones en este ámbito. Se concluye que Barcelona tiene el reto de asumir el paso de la gestión del turismo (modelo de gestión de la promoción y fomento del turismo) a la gestión de la ciudad (turística), con la adecuación del organigrama del gobierno de la ciudad a la nueva realidad. En los procesos de gestión de este nuevo modelo de ciudad se tendrán en cuenta los nuevos actores, sus intereses, sus aportaciones. Este nuevo escenario requiere la implantación de la cultura de la negociación y el acuerdo para hacer compatibles los diversos intereses y las diferentes funciones de la ciudad, y minimizar las afectaciones no deseadas y el conflicto.

\section{c) Ciudad turística, destino Barcelona}

Los procesos de producción/consumo turísticos y de construcción de la ciudad están cambiando de escala y dibujan una nueva situación: la proyección espacial del turismo está alcanzando una nueva dimensión física y funcional. La ciudad turística rebasa los límites de la ciudad administrativa, reflejo de la Barcelona real (la gran Barcelona), que ha superado los límites del municipio. La "gran ciudad" turística tiene unos límites imprecisos pero se difunde por el litoral y por el interior. Conceptualmente constituye el "Destino Barcelona». En las coordenadas de la gestión pública del turismo surgen nuevos retos debido a la disfunción entre los ámbitos espaciales de la funcionalidad del turismo y los ámbitos espaciales político-administrativos (municipios): la gestión del turismo ha de adaptarse a la nueva realidad y, si es necesario, adoptar nuevos objetivos, nuevos medios y herramientas y un nuevo modelo organizativo.

\section{d) Crecimiento, desarrollo, consolidación, saturación, ¿crisis?}

¿Hasta qué punto es aplicable a Barcelona la teoría del ciclo de vida del producto-destino? ¿Es adecuado aplicarlo al caso de Barcelona, ante la complejidad de elementos y la diversidad de funciones que la caracterizan, tal como ocurre con otras grandes ciudades? Barcelona (o la "gran ciudad turística»), como cualquier otro "producto-destino» turístico, está sometida a la sucesión de fases de comportamiento del mercado turístico y, por tanto, sus actores deben saber gestionar el sistema turístico y la ciudad como destino turístico tanto en períodos de crecimiento como en períodos de estancamiento o de pérdida de atractivo, ya sea en ciclos de expansión económica o de crisis. Ante las oscilaciones del mercado turístico, Barcelona es menos vulnerable que las ciudades especializadas exclusivamente en turismo; y ello se debe a dos razones: a su perfil de ciudad global, con diversas funciones estructurales, y, por otra parte, al hecho de que en Barcelona conviven diversos turismos, diversos productos específicos. 


\section{Referencias bibliográficas}

Ajuntament de Barcelona (2010). Pla Estratègic de Turisme de la Ciutat de Barcelona 2015. Barcelona: Ajuntament de Barcelona-Turisme de Barcelona. Comprende diversos documentos básicos y estudios específicos que pueden consultarse en la página web <http://www.turismebcn2015.cat>.

André Romero, M. ${ }^{a}$ Encarnación (1999). El turismo urbano y la segmentación motivacional. Aplicación econométrica a la ciudad de Barcelona. Barcelona: Universitat de Barcelona. Departament d'Econometria, Estadística i Economia Espanyola. Tesis doctoral. <http://www.tdx.cat/handle/10803/43>

AQR-IREA (2009). Impacte econòmic de l'activitat turística a la ciutat de Barcelona, 2007. Barcelona: Pla Estratègic de Turisme de la Ciutat de Barcelona. Estudis.

Benach, Núria (1993). «Producción de imagen en la Barcelona del 92». Estudios Geográficos, vol. LIV, núm. 212, 483-506.

Bonilla Bolaños, Diana (2014). Estudi i valoració del Pla Estratègic de Turisme de la Ciutat de Barcelona 2015. Barcelona: Universitat de Barcelona. Trabajo final del máster de Planificación Territorial y Gestión Ambiental.

Capel, Horacio (2005). Modelo Barcelona: un examen critico. Barcelona: Serbal.

Delàs, Ignacio de (1995). «El turismo metropolitano en Europa. Una iniciativa innovadora de promoción turística local: el caso de Barcelona». Estudios Turísticos, 126: 151-160.

Delgado, Manuel (2007). La ciudad mentirosa: fraude y miseria del «modelo Barcelona». Barcelona: Los Libros de la Catarata.

Duran, Pere (2004). «El turisme, una realitat estratègica». Barcelona. Metròpolis mediterrània, 5, 121-125.

European Cities Marketing (2014). The European Cities Marketing. Benchmarking Report (10 Official Edition 2013-2014). <www.europeancitiesmarketing.com>

Font Garolera, Jaume y Sánchez Aguilera, Dolores (2014). «Aproximación a un modelo de turismo urbano de éxito: la Barcelona postolímpica». En: López Palomeque, F. y Cànoves, G. (eds.). Turismo y territorio. Innovación, renovación $y$ desafios. Valencia: Tirant lo Blanch, 63-176.

Garay Tamajón, Luis Alfonso y Cànoves Valiente, Gemma (2012), «Turismo de cruceros en Barcelona. De la marginalidad al liderazgo internacional». Boletín de la Asociación de Geógrafos Españoles, 60, 253-271.

Garcés, Marina (2014). «Desmarcar Barcelona». Conferencia pronunciada el día 23 de mayo de 2014 en el Centre de Cultura Contemporánea de Barcelona (CCCB).

García García de León, Aurora (2013). Branding de Ciudad: la promoción del modelo Barcelona y su proyección como marca. Barcelona: Departament de Composició Arquitectònica, Universitat Politècnica de Catalunya. Tesis doctoral. <http://www.tdx.cat/handle/10803/129517>

Gausa, Manuel; Banchini, Silvia y Falcón, Luis (2010). MultiRamblas. La Barcelona Mar. BCN 6T. Territorio - Turismo - Tiempo - Tecnología - Talento - Tolerancia. Barcelona: Actar.

López Palomeque, Francisco (1995). «La estrategia del turismo metropolitano: el caso de Barcelona». Estudios Turísticos, 126, 119-141.

- (2002). "Turisme urbà i gestió pública: Barcelona com a escenari turístic». En: Tello, R. (coord.). Espais públics. Mirades multidisciplinars. Barcelona: Pòrtic, $163-185$. 
- (2009). «Barcelona, ciutat turística». En: López Palomeque, F. y SÁnchez AguiLERA, D. (dirs.). Atles del turisme a Catalunya. Mapa nacional de l'oferta i els productes turistics. Barcelona: Departament d'Innovació, Universitats i Empresa. Generalitat de Catalunya, 442-451. (Versión web: http://www.atlesturismecatalunya.cat/).

Oppenheimer, Walter (2015). «La doble vida de Barcelona». El País (5 de febrero), Quadern, 1-3.

Palou Rubio, Saida (2010). Barcelona, destinació turística. Promoció pública, turismes, imatges i ciutat (1988-2010). Barcelona: Departament d'Antropologia Cultural i Història d'Amèrica i d'Àfrica. Universitat de Barcelona. Tesis doctoral. <http://www.tesisenxarxa.net/handle/10803/21771>

- (2012). Barcelona, destinació turística. Un segle d’imatges i promoció pública. Barcelona: Vitel.la. Turisme Cultural.

Serra, Joan (2010). «Síntesis del Plan Estratégico de Turismo de la Ciudad de Barcelona 2015». En: Dinámicas de transformación del turismo en el S. XXI. Actas del XV Congreso AECIT-Tenerife (Islas Canarias, 24, 25 y 26 noviembre 2010).

Serrano Miracle, Damià y Figueroa Domeneq, Cristina (2014). «Las dos grandes urbes: Madrid y Barcelona». En: García Sánchez, A. (coord.). 20 años de la actividad turística en España. Madrid: AECIT-Síntesis, 183-195.

Turisme de Barcelona (2014). Estadistiques de turisme de Barcelona, 2013. Barcelona: Turisme de Barcelona.

Vera, J. Fernando; López Palomeque, Francisco; Marchena, Manuel J. y Anton, Salvador (2013). Análisis territorial del turismo y planificación de destinos turísticos. Valencia: Tirant lo Blanch. 